\title{
Growth-preserving instrumentation in early-onset scoliosis patients with multi-level congenital anomalies
}

\author{
R. Carter Clement ${ }^{1} \cdot$ Burt Yaszay $^{2}$ (D) Anna McClung ${ }^{3} \cdot$ Carrie E. Bartley $^{2} \cdot$ Naveed Nabizadeh $^{2} \cdot$ David L. Skaggs $^{4}$. \\ George H. Thompson ${ }^{5}$. Ohenaba Boachie-Adjei ${ }^{6}$. Paul D. Sponseller ${ }^{7}$. Suken A. Shah ${ }^{8}$. James O. Sanders ${ }^{9}$. \\ Jeff Pawelek ${ }^{3}$. Gregory M. Mundis ${ }^{1}$. Behrooz A. Akbarnia ${ }^{10}$. Pediatric Spine Study Group (Formerly, Growing Spine \\ Study Group) ${ }^{3}$
}

Received: 22 March 2021 / Accepted: 31 May 2021 / Published online: 21 June 2021

(c) Scoliosis Research Society 2021

Dear Editor,

We appreciate the interest in our article by the letter authors. The authors suggest this is a very complex patient population. While they fall under a single diagnosis, congenital early-onset scoliosis, the patient population still is very heterogenous. As described in the paper, even with placement of GPI, there was significant variability in the amount of initial correction with nearly one third not obtaining any real significant correction. This compares to about a quarter of patients obtaining greater than $50 \%$ correction. This

This reply refers to the comment available online at https://doi.org/ 10.1007/s43390-020-00249-4.

Burt Yaszay

byaszay.rady@gmail.com

1 Department of Orthopaedics, Children's Hospital of New Orleans, New Orleans, LA, USA

2 Department of Orthopedics, Rady Children's Hospital, San Diego, CA, USA

3 Growing Spine Foundation, Milwaukee, WI, USA

4 Children's Orthopedic Center, Children's Hospital Los Angeles, Los Angeles, CA, USA

5 Rainbow Babies and Childrens Hospital, Case Western Reserve University, Cleveland, OH, USA

6 Focos Orthopaedic Hospital, Accra, Ghana

7 Department of Orthopaedic Surgery, The Johns Hopkins University, Baltimore, MD, USA

8 Nemours/Alfred I. duPont Hospital for Children, Wilmington, DE, USA

9 Department of Orthopedics, University of North Carolina, Chapel Hill, NC, USA

10 Department of Orthopeadics, University of California, San Diego, San Diego, CA, USA would suggest variability in the degree of open flexible discs and, therefore, some difficulty in evaluating specific effects for the entire cohort during the GPI interval. We agree further insight could be gained if, for example, we studied the populations based on this initial correction. Since there were limited numbers of patients in each group, this was not performed. Another study could be performed with a larger cohort. This would also benefit from pre-index flexibility films which were not readily available in our patients.

As for the concern regarding the influence of the final follow-up, we feel that this is an important part of the study. The final fusion is part of the treatment process and we believe that it is an important data point for patients with EOS. Extrapolating this work with other studies that show shorter-term follow-up can be misleading and should not be generalized to this study. The concept of diminishing returns and risk of spontaneous fusion with prolonged treatment, while not evaluated in this study, would suggest that studies that do not evaluate patients to final fusion (or equivalent) are not evaluating the full treatment.

Finally, we are glad that the authors are looking at other methods/devices of GPI. Our purpose was not to compare different forms of GPI. Most of our patients were managed with traditional growing rods. We never intended to suggest best treatment practices but only to demonstrate the results of the treatment utilized during this era of EOS management.

Thank you.

Burt Yaszay, MD on behalf of all coauthors.

Funding No external funding was received.

Publisher's Note Springer Nature remains neutral with regard to jurisdictional claims in published maps and institutional affiliations. 